\title{
Anestesia com fluxo baixo de gases frescos em cães
}

\author{
Fresh gas low flow anesthesia in dogs
}

Danielli Parrilha de Paula ${ }^{1}$ Newton Nunes ${ }^{2 *}$

\section{Resumo}

Com este trabalho, os autores procuram fornecer informações sobre a anestesia com fluxo baixo em cães. São tecidas considerações relativas ao emprego da técnica, suas vantagens e restrições de uso, bem como a avaliação comparativa dos anestésicos voláteis quanto à sua eficiência e segurança quando utilizados sob anestesia com fluxo baixo de gases frescos. Complementarmente, procurou-se atualizar com literatura recente os conhecimentos dos profissionais que militam na área de Anestesiologia Veterinária.

Palavras-chave: Anestesia, baixo fluxo, cães.

\begin{abstract}
With this work, the authors provide information about low flow anesthesia in dogs. They comment about the use of this technique, its advantages and disadvantages, and a comparative evaluation about volatile anesthetics regarding to their efficiency and safety when used in low flow anesthesia. Complementary, it was aimed to update the professionals that work with Veterinary Anesthesiology, through new papers and reports.
\end{abstract}

Key words: Anesthesia, low flow, dogs.

\section{Introdução}

O desenvolvimento de novos agentes e técnicas anestésicas representa um grande avanço para a Anestesiologia Veterinária e possibilita ao anestesiologista procedimentos mais seguros minimizando os riscos inerentes aos procedimentos anestésicos.

Uma nova técnica é a anestesia com fluxo baixo, que está ganhando popularidade por diversos motivos. Menor consumo anestésico, portanto custo baixo, são razões que favorecem o uso desta técnica (SIEBER et al., 2000). Outra vantagem de seu uso é a redução da poluição ambiental, já que o baixo fluxo de gases frescos ajuda a reduzir a emissão de clorofluorocarbonos halogenados (SUTTNER; BOLDT, 2000).

Esta técnica tem ganhado mais atenção dos anestesistas, e tem sido cada vez mais estudada a fim de elucidar e controlar todos os riscos inerentes a sua utilização.

\section{Técnica}

A técnica descrita por Wagner e Bednarski (1992), Brock (1995) e Concannon (1996) cita que, após indução e intubação do animal, este é conectado

\footnotetext{
${ }^{1}$ Doutoranda do Programa de Pós-graduação em Cirurgia Veterinária /FCAV/UNESP/Campus de Jaboticabal-SP

${ }^{2}$ Professor Assistente Doutor, Departamento de Clínica e Cirurgia Veterinária/FCAV/UNESP/Campus de Jaboticabal-SP - CEP: 14884-900, Jaboticabal, SP. E-mail: newton@ fcav.unesp.br

* Autor para correspondência.
} 
Paula, D. P. de; Nunes, N.

ao circuito anestésico e primeiramente mantido num fluxo de oxigênio de 50 a $200 \mathrm{~mL} / \mathrm{kg} / \mathrm{min}$ durante 15 a 20 minutos. Este fluxo alto é necessário para adequar a quantidade ideal de gases anestésicos para manter uma anestesia cirúrgica e garantir saturação adequada de $\mathrm{O}_{2}$ na mistura de gases. Em seguida, o fluxo de $\mathrm{O}_{2}$ pode ser diminuído para valores iguais ao requerimento metabólico (4 a $7 \mathrm{~mL} / \mathrm{kg} / \mathrm{min}$ ) e, simultaneamente, a válvula de alívio é fechada.

Durante a anestesia com fluxo baixo, é difícil ocorrerem mudanças bruscas no plano anestésico. Para que tal situação aconteça, é necessário que se aumente o fluxo de $\mathrm{O}_{2}$, abra a válvula de alívio e esvazie o balão (CONCANNON, 1996).

\section{Menor Desperdício de Gases Frescos}

Segundo Wagner e Bednarski (1992), os Médicos Veterinários realizam anestesia em pequenos animais utilizando fluxo de oxigênio de 22 a $44 \mathrm{~mL} / \mathrm{kg} / \mathrm{min}$, ou um fluxo de 1,2 ou $3 \mathrm{~L} / \mathrm{min}$, baseados em suas preferências pessoais e hábito. Considerando-se que o requerimento metabólico dos cães e gatos é de 4 a $7 \mathrm{~mL} / \mathrm{kg} / \mathrm{min}$, há desperdício de grande parte do oxigênio. Juntamente com o oxigênio, também é perdido o anestésico volátil utilizado, acarretando em um maior consumo de anestésico.

Suttner et al. (2000) relata que, dependendo do sistema de ventilação utilizado, até $90 \%$ do anestésico administrado é eliminado para a atmosfera sem uso. Com a prática de fluxo baixo a maior parte dos gases expirados são reciclados e retornam aos pulmões do paciente após o dióxido de carbono ter sido absorvido, diminuindo assim, o consumo de gases frescos (COTTER et al., 1991; SUTTNER et al., 2000).

No sistema de fluxo alto o $\mathrm{CO}_{2}$ é diluído e dispersado nos gases frescos, o que acarreta em maior aporte desses gases, consequentemente, maior desperdício (WHITE, 1999).

\section{Menor Poluição Ambiental}

Atualmente, a questão da poluição ambiental vem sendo tema de discussão no mundo inteiro. Nesse contexto, o baixo fluxo de gases frescos ganha o mérito de diminuir a poluição do meio ambiente, já que esta técnica ajuda a reduzir a emissão de clorofluorocarbonos halogenados (SUTTNER; BOLDT, 2000). A menor emissão desses compostos no ambiente de trabalho também é um ponto que deve ser lembrado pois, principalmente os anestesistas que ficam muito tempo ao lado do aparelho de anestesia, acabam inalando esses compostos, que ao longo do tempo podem provocar danos à sua saúde.

\section{Umidificação e Aquecimento dos Gases Inspirados}

Aquecimento e umidificação dos gases inspirados são características observadas com o uso de fluxo baixo (ALDRETE; CUBILLOS; SHERRILL, 1981; BERNTMAN; LUTTROPP; WERNE, 1990; COTTER et al., 1991; CONCANNON, 1996; BEAMS et al., 1998; JOHANSSON; LUNDBERG; LUTTROPP, 2001). Essas características são importantes para manutenção da temperatura corpórea durante o período em que o paciente permanece anestesiado. Esse aquecimento dos gases pode ser de até $6{ }^{\circ} \mathrm{C}$ (BAXTER, 1997).

Uma inalação prolongada de gases anestésicos secos pode resultar num prejuízo da função do epitélio ciliado do trato respiratório, induzir a reações inflamatórias, predispor atelectasias ou pneumonia e diminuir a temperatura corpórea (SUTTNER; BOLDT, 2000). A anestesia com fluxo baixo contribui significativamente para a conservação do calor e umidade do trato respiratório, dispensando o uso de equipamentos para aquecimento e umidificação dos gases (BENGTSON; SONANDER; STENQVIST, 1987; KLEEMANN, 1994). 


\section{Anestésicos Voláteis}

Estudos comparando o desfluorano ao sevofluorano e enflurano, no homem, permitiram concluir que o desfluorano oferece significantes vantagens para a manutenção da anestesia com fluxo baixo sobre os outros dois anestésicos. Ele provoca depressão cardiovascular mínima, recuperação anestésica mais rápida e mantém melhor a pressão arterial dos pacientes (XIE; JIANG, 1997). Contudo, Muir e Gadawski (1998) concluíram que a utilização do sevofluorano em sistema de fluxo baixo em cães, não ocasionou depressão cardiorrespiratória significativa. Baum et al. (1997) afirmaram que, devido à sua baixa solubilidade e reduzida biotransformação, o desfluorano é apropriado para o uso em anestesias com fluxo baixo. Ebert et al. (1999) descreveram que tanto o desfluorano como o sevofluorano têm efeitos semelhantes quando usados sob fluxo baixo.

Anestésicos como sevofluorano e desfluorano, que têm baixa solubilidade sangue/gás, são opções para uso com fluxo baixo. $\mathrm{O}$ emprego destes agentes pode ser otimizado já que o custo operacional é menor com essa técnica (SUTTNER; BOLDT, 2000). O isofluorano também vem sendo amplamente utilizado por ter características como alta potência anestésica e ínfima taxa de metabolização $(0,2 \%$ aproximadamente).

Estudos utilizando o halotano em anestesia com fluxo baixo são raros, isso pode ser interpretado pelo fato deste agente promover efeitos indesejáveis como, sensibilização do miocárdio às catecolaminas, elevação da pressão intracraniana, diminuição da pressão arterial, probabilidade de provocar hipertermia maligna; além disso, este agente vem sendo cada vez menos utilizado na prática anestésica, já que outros anestésicos voláteis, mais potentes e seguros, estão disponíveis no mercado.

\section{Formação do Composto A}

Sabe-se que o sevofluorano, na presença de absorventes de $\mathrm{CO}_{2}$, gera produtos de degradação potencialmente tóxicos (LIU et al., 1991; FRINK et al., 1992). Cinco destes produtos foram identificados in vitro, sendo que o composto A foi classificado como possível causador de toxicidade renal (BITO; IKEDA, 1994a). A concentração desses produtos aumenta quando a temperatura dos absorventes de $\mathrm{CO}_{2}$ é alta (BITO; IKEDA, 1994b). De acordo com Frink et al. (1992), quando é utilizada a cal baritada como absorvente de $\mathrm{CO}_{2}$, a concentração do composto A é 2,5 vezes maior do que quando é usada a cal sodada; sua concentração é maior também quando utilizada a anestesia com fluxo baixo (BITO; IKEDA, 1994b). Porém há estudos mostrando que a quantidade de composto A formado pela degradação do sevofluorano pelos absorventes de $\mathrm{CO}_{2}$, não é significativamente aumentada durante a anestesia com fluxo baixo (EBERT et al., 1998; MUIR; GADAWSKI, 1998).

Estudos vêm sendo realizados a fim de elucidar os riscos inerentes ao uso do sevofluorano sob fluxo baixo. Okada et al. (1997) utilizaram o sevofluorano e óxido nitroso para manutenção da anestesia durante 5 horas no homem e concluíram que a concentração do composto A produzido independe da duração da anestesia. Obata et al. (2000) também descreveram que a anestesia prolongada com fluxo baixo utilizando sevofluorano não produz disfunção renal ou hepática.

Sun et al. (1997) estudaram os efeitos provocados pela repetição da anestesia com fluxo baixo em cães. Os animais foram mantidos anestesiados com sevofluorano por 6 horas, sendo repetido o procedimento após 7 dias. Foi mensurada a quantidade de composto A produzido e chegou-se a conclusão que a repetição da anestesia utilizando-se fluxo baixo com sevofluorano não provocou alterações significantivas nas funções hepática e renal. Outro estudo em cães foi realizado por Suzuki et al. (1995), onde Beagles foram mantidos anestesiados por 48 horas, com fluxo baixo de gases, utilizando o sevofluorano como agente inalatório. A histopatologia hepato-renal mostrou alterações similares às observadas em cães anestesiados com isofluorano e alto fluxo de gases. 
Bito e Ikeda (1996) compararam a produção do composto A quando utilizada a anestesia com fluxo baixo com sevofluorano e isofluorano e anestesia em fluxo alto com sevofluorano concluindo que a função renal pós-anestésica é a mesma para os três tipos de anestesia.

A anestesia com fluxo baixo utilizando desfluorano ou sevofluorano em pacientes idosos praticamente não afetou a integridade hepática (SUTTNER et al., 2000). Outrossim, não há evidências que esta técnica utilizando o desfluorano (BAUM et al., 1997) ou isofluorano (MORIMOTO; TAMURA; MATSUMOTO, 1998) venha a ser responsável pelo aumento do risco de envenenamento acidental pelo monóxido de carbono.

\section{Aparelhos de Anestesia para Uso com Fluxo Baixo de Gases}

Igarashi et al. (1993) testaram um aparelho para uso durante anestesias com fluxo baixo - o Cícero. Este aparelho é equipado com monitor de concentração de $\mathrm{O}_{2}$, agentes anestésicos e $\mathrm{CO}_{2}$ no circuito anestésico, e vaporizador calibrado para concentração do agente. Apresenta um mecanismo de absorção de $\mathrm{CO}_{2}$ onde o gás expirado passa três vezes pelo caníster. A temperatura da cal sodada e umidade no circuito também são aferidas, bem como o consumo anestésico tanto para fluxo baixo como alto. Apesar de observarem que a temperatura da cal sodada é maior quando utilizado o fluxo baixo, este aparelho mostrou-se seguro e econômico. Porém, os aparelhos de anestesia usados atualmente podem ser utilizados com bons resultados, desde que haja a monitoração adequada dos pacientes.

\section{Monitoração e Restrições de Uso}

Como qualquer outra técnica anestésica, o fluxo baixo tem contra-indicações e os anestesistas devem conhecer os riscos e limitações associadas ao seu uso. Durante este tipo de anestesia deve-se monitorar atentamente a capnometria e a oximetria a fim de não permitir a instalação de um quadro de hipóxia. A realização de hemogasometria é de extrema valia para a avaliação do estado ventilatório dos pacientes durante a anestesia com fluxo baixo de oxigênio, permitindo identificar precocemente qualquer alteração na oxigenação, pois hipóxia acidental, hipercapnia, plano anestésico inadequado e acúmulo de gases potencialmente tóxicos, são riscos associados ao uso da técnica. Portanto, o conhecimento básico da distribuição dos gases anestésicos e uma monitoração adequada da oximetria, capnometria, oxigênio inspirado, concentração de gases e o emprego de fluxômetro de precisão são fundamentais para a segurança de uma anestesia com baixo fluxo diluente (SUTTNER et al., 2000).

É possível o uso de ventilação controlada desde que seja adotada a ventilação no modo tempo controlado, pois assim pode-se manter o baixo fluxo de gases frescos.

Não deve ser utilizado fluxo baixo de oxigênio quando for utilizado o sistema de Bain ou outros circuitos onde não há reinalação de gases. Nesses casos o alto fluxo de $\mathrm{O}_{2}$ é necessário, pois o sistema é desprovido de caníster com cal soldada para a remoção do dióxido de carbono expirado (WAGNER; BEDNARSKI, 1992; BROCK, 1995).

\section{Conclusão}

A anestesia com fluxo baixo é um método simples, porém muito efetivo para minimizar custos e pode ser aplicado a um grande número de pacientes, sem comprometer sua saúde ou segurança (SUTTNER; BOLDT, 2000).

Os anestesiologistas têm um compromisso moral e ético de minimizar o custo de procedimentos anestésicos, sem contudo, pôr em risco a segurança de seus pacientes. A anestesia com fluxo baixo vem ganhando cada vez mais espaço por conseguir unir segurança e menores gastos, além disso ainda reduz a poluição ambiental. 


\section{Referências}

ALDRETE, J. A.; CUBILLOS, P.; SHERRILL, D. Humidity and temperature changes during low flow and closed system anaesthesia. Acta Anaesthesiologica Scandinavica, Copenhagen, v.25, p.312-14, 1981.

BAUM，J.; BERGHOFF，M.; STANKE，H. G.; PETERMEYER, M.; KALFF, G. Low-flow anesthesia with desflurane. Anaesthesist, Berlin, v.46, p.287-93, 1997.

BAXTER, A. D. Low and minimal flow inhalational anaesthesia. Canadian Journal of Anaesthesia, Ontario, v.44, p.643-53, 1997.

BEAMS, D. M.; SASSE, F. J.; WEBSTER, J. G.; RADWIN, R. G. Model for the administration of low-flow anaesthesia. British Journal of Anaesthesia, Oxford, v.81, p.161-70, 1998.

BENGTSON, J. P.; SONANDER, H.; STENQVIST, O. Preservation of humidity and heat of respiratory gases during anaesthesia: a laboratory investigation. Acta Anaesthesiologica Scandinavica, Copenhagen, v.31, p.127-31, 1987.

BERNTMAN, L; LUTTROPP, H. H.; WERNER, O. Mechanical ventilation during low-flow anaesthesia. Anaesthesia, London, v.45, p.855-58, 1990.

BITO, H.; IKEDA, K. Closed-circuit anesthesia with sevoflurane in humans: effects on renal and hepatic function and concentrations of breakdown products with soda lime in the circuit. Anesthesiology, Philadelphia, v.80, p.71-6, 1994b.

BITO, H.; IKEDA, K. Long-duration, low-flow sevoflurane anesthesia using two carbon dioxide absorbantsQuantification of degradation products in the circuit. Anesthesiology, Philadelphia, v.81, p.340-45, 1994a.

BITO, H.; IKEUCHI, Y.; IKEDA, K. The effects of lowflow sevoflurane anesthesia on renal function. Anesthesiology, Philadelphia, v.85, p.87-91, 1996.

BROCK, N. Low flow anesthesia revised. Canadian Veterinary Journal, Ottawa, v.36, p.366-7, 1995.

CONCANNON, K. T. Using low-flow anesthesia. Veterinarni Medicina, Praha, v.4, p.349-52, 1996.

COTTER, S. M.; PETROS, A J.; DORE; C. J.; BARBER, N. D.; WHITE, D. C. Low-flow anaesthesia. Anaesthesia, London, v.46, p.1009-12, 1991.

EBERT, T. J.; MESSANA, L. D.; UHRICH, T. D.; STAACKE, T. S. Absence of renal and hepatic toxicity after four hours of 1.25 minimum alveolar anesthetic concentration sevoflurane anesthesia in volunteers. Anesthesia \& Analgesia, Baltimore, v.86, p.662-7, 1998.
FRINK, E. J.; MALAN, T. P.; MORGAN, S. E.; BROWN, E. A.; MALCOMSON, M.; BROWN, B. R.. Quantification of the degradation products of sevoflurane in two $\mathrm{CO}_{2}$ absorbants during low-flow anesthesia in surgical patients. Anesthesiology, Philadelphia, v.77, p.1064-1069, 1992.

IGARASHI, H.; WATANABE, H.; IWASAKI, H.; MAEDA, T.; NAMIKI, A. Low-flow anesthesia with a newly developed anesthetic machine, Cicero. Anesthesiology, Philadelphia, v.79, p.458-63, 1993.

JOHANSSON, A.; LUNDBERG, D.; LUTTROPP,H.H. Lowflow anaesthesia with desflurane: kinetics during clinical procedures. European Journal of Anaesthesiology, Oxford, v.18, p.499-504, 2001.

KLEEMANN, P. P. Humidity of anaesthetic gases with respect to low-flow anaesthesia. Anaesthesia and Intensive Care, Sydney, v.22, p.127-31, 1994.

LIU, J.; LASTER, M.J.; EGER, E.I.; TAHERIL, S. Absorption and degradation of sevoflurane and isoflurane in a conventional anesthetic circuit. Anesthesia \& Analgesia, Baltimore, v.72, p.785-98, 1991.

MORIMOTO, Y.; TAMURA, T.; MATSUMOTO, S. Carbon monoxide concentrations during low flow anesthesia. Masui: The Japanese Journal of Anesthesiology, Morioka,, v.47, n.1, p.90-3, 1998.

MUIR, W. W.; GADAWSKI, J. Cardorespiratory effetcs of low-flow and closed circuit inhalation anesthesia, using sevoflurane delivered with na in-circuit vaporizer and concentrations of compound A. American Journal of Veterinary Research, Schaumburg, v.59, p.603-8, 1998.

OBATA, R.; BITO, H.; OHMURA, M.; MORIWAKI, G.; IKEUCHI, Y.; KATOH, T.; SATO, S. The efects of prolonged low-flow sevoflurane anesthesia on renal and hepatic function. Anesthesia and Analgesia, Baltimore, v.91, p.1262-8, 2000.

OKADA, K.; ASANO, N; KIMURA, O.; OKADA, H.; NISHIO, S.; WAKUSAWA, R.L. Low-flow anesthesia using a fresh gas flow of $600 \mathrm{~mL}$.min-1 for 5 hours. Masui: The Japanese Journal of Anesthesiology, Morioka, v.46, n.10, p.1321-8, 1997.

SIEBER, T. J.; FREI, C. W.; DERIGHETTI, M.; FEIGENWINTER, P.; LEIBUNDGUT, D. ZBINDEN, A. M. Model-based automatic feedback control versus human control of end-tidal isoflurane concentration using lowflow anaesthesia. British Journal of Anaesthesia, Oxford, v. 85, n.6, p.818-25, 2000.

SUN, L.; SUZUKI, Y.; TAKATA, M.; MIYASAKA, K. Repeated low-flow sevoflurane anesthesia: effects on hepatic and renal function in beagles. Masui: The Japanese Journal of Anesthesiology, Morioka, v.46, n.3, p.351-357, 1997. 
Paula, D. P. de; Nunes, N.

SUTTNER, S. W.; BOLDT, J. Low-flow anaesthesia: does it have potential pharmacoeconomic consequences? PharmacoEconomics, Auckland, v.17, n.6, p.585-590, 2000.

SUTTNER, S. W.; SCHMIDT, C. C.; BOLDT, J.; HUTTNER, I., KUMLE, B.; PIPER, S. N. Low-flow desflurane and sevoflurane anesthesia minimaly affect hepatic integrity and function in elderly patients. Anesthesia \& Analgesia, Baltimore, v.91, n.1, p.206-12, 2000.

SUZUKI, Y.; SUN, L.; TAKATA, M.; MIYASAKA, K. Effects of prolonged sevoflurane and isoflurane low-flow anesthesia: histhopatological study after 48 hours anesthesia in Beagles. Anesthesiology, Philadelphia, v.83, p. 254-59, 1995.
WAGNER, A. E.; BEDNARSKI, R. M. Use of low-flow and closed-system anesthesia. Journal of the American Veterinary Medical Association, Schaumburg, v.200, p.1005-10, 1992.

WHITE, D. C. The history and development of low-flow breathing system souce. In: IEE SEMINAR: LOW-FLOW ANAESTHESIA BREATHING SYSTEM-TECNOLOGY, London, 1999. Proceeding. London: IEEE, 1999. p.1

XIE, G.; JIANG, H. Clinical study of desflurane on lowflow anesthesia compared with sevoflurane and enflurane. Chinese Medical Journal, Peking, v.110, p.707-10, 1997. 